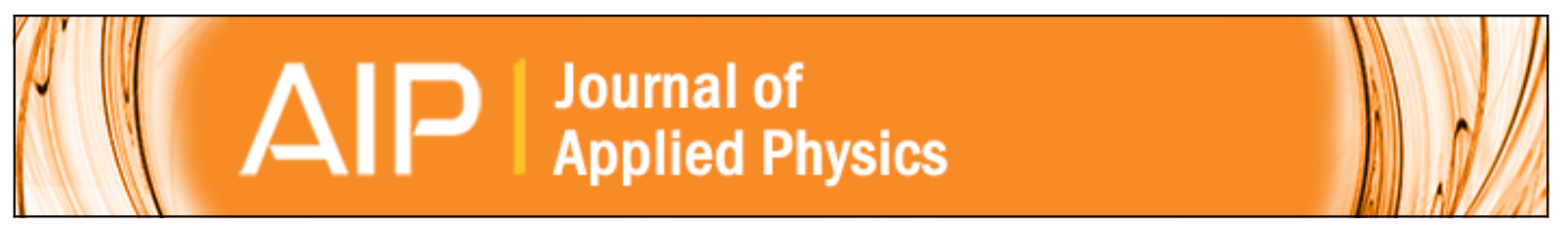

\title{
Study of the relation between oxygen vacancies and ferromagnetism in Fe-doped TiO2 nano-powders
}

Azucena M. Mudarra Navarro, Claudia E. Rodríguez Torres, Vitaliy Bilovol, A. Fabiana Cabrera, L. A. Errico, and M. Weissmann

Citation: Journal of Applied Physics 115, 223908 (2014); doi: 10.1063/1.4883183

View online: http://dx.doi.org/10.1063/1.4883183

View Table of Contents: http://scitation.aip.org/content/aip/journal/jap/115/22?ver=pdfcov

Published by the AIP Publishing

\section{Articles you may be interested in}

Room temperature ferromagnetism in defective $\mathrm{TiO} 2$ nanoparticles: Role of surface and grain boundary oxygen vacancies

J. Appl. Phys. 114, 203906 (2013); 10.1063/1.4833562

Long-range ferromagnetic order induced by a donor impurity band exchange in $\mathrm{SnO}$ :Er3+ nanoparticles

J. Appl. Phys. 114, 203902 (2013); 10.1063/1.4833549

Structural, optical, vibrational, and magnetic properties of sol-gel derived Ni doped $\mathrm{ZnO}$ nanoparticles

J. Appl. Phys. 114, 033912 (2013); 10.1063/1.4813868

Room temperature ferromagnetism in non-magnetic doped TiO2 nanoparticles

J. Appl. Phys. 113, 17B511 (2013); 10.1063/1.4795615

Correlation between oxygen vacancies and magnetism in Mn-doped Y2O3 nanocrystals investigated by defect engineering techniques

Appl. Phys. Lett. 101, 022408 (2012); 10.1063/1.4732094

\section{AP $\mid \begin{aligned} & \text { Journal of } \\ & \text { Applied Physics }\end{aligned}$}

Journal of Applied Physics is pleased to announce André Anders as its new Editor-in-Chief 


\title{
Study of the relation between oxygen vacancies and ferromagnetism in Fe-doped $\mathrm{TiO}_{2}$ nano-powders
}

\author{
Azucena M. Mudarra Navarro, ${ }^{1}$ Claudia E. Rodríguez Torres, ${ }^{1, a)}$ Vitaliy Bilovol, ${ }^{2}$ \\ A. Fabiana Cabrera, ${ }^{1}$ L. A. Errico, ${ }^{1,3}$ and M. Weissmann ${ }^{4}$ \\ ${ }^{1}$ Departamento de Física - Facultad de Ciencias Exactas, UNLP, Instituto de Física La Plata, CCT-La Plata, \\ CONICET, CC 67, 1900 La Plata, Argentina \\ ${ }^{2}$ Laboratorio de Sólidos Amorfos, INTECIN-CONICET, Facultad de Ingeniería, UBA, Av. Paseo Colón 850, \\ Buenos Aires C1063ACV, Argentina \\ ${ }^{3}$ Universidad Nacional del Noroeste de la Pcia. de Buenos Aires (UNNOBA), Monteagudo 2772, \\ Pergamino. CP 2700 Buenos Aires, Argentina \\ ${ }^{4}$ Depto. de Física, Comisión Nacional de Energía Atómica, Av. del Libertador 8250, 1429 Buenos Aires, \\ Argentina
}

(Received 6 January 2014; accepted 2 June 2014; published online 11 June 2014)

\begin{abstract}
In this work, we present an experimental and theoretical study of structural and magnetic properties of $\mathrm{Fe}$ doped rutile $\mathrm{TiO}_{2}$ nanopowders. We show that Fe-doping induces the formation of oxygen vacancies in the first-sphere coordination of iron ions, which are in +2 and +3 oxidation states. We found that $\mathrm{Fe}$ ions form dimers that share one oxygen vacancy in the case of $\mathrm{Fe}^{3+}$ and two oxygen vacancies in the case of $\mathrm{Fe}^{2+}$. The saturation magnetization is almost independent of iron concentration and slightly increases with the relative fraction of $\mathrm{Fe}^{2+}$. Ab initio calculations show that two Fe ions sharing an oxygen vacancy are coupled ferromagnetically, forming a bound magnetic polaron (BMP), but two neighbor BMPs are aligned antiparallel to each other. Extra electron doping plays a fundamental role mediating the magnetic coupling between the ferromagnetic entities: carriers, possibly concentrated at grain boundaries, mediate between the BMP to produce ferromagnetic alignment. (C) 2014 AIP Publishing LLC. [http://dx.doi.org/10.1063/1.4883183]
\end{abstract}

\section{INTRODUCTION}

Magnetism in wide-gap semiconductor oxides doped with $3 d$ transition elements, usually called dilute magnetic semiconductor oxides (O-DMS), has become one of the most interesting topics in condensed matter physics. New experimental and theoretical publications show significant advances in the synthesis and characterization methods, ${ }^{1-5}$ but the microscopic mechanism of long-range magnetic order is still an open problem.

Most of the research efforts in this field were focused on Co- and Fe-doped $\mathrm{TiO}_{2}, \mathrm{SnO}_{2}, \mathrm{ZnO}$, and $\mathrm{In}_{2} \mathrm{O}_{3}$. There is consensus in the scientific community that an important role is played by defects, especially oxygen vacancies, for the occurrence of ferromagnetic interactions. ${ }^{6-10}$ Except in few cases (such as Refs. 11 and 12), bulk samples and thin films of well crystallized and stoichiometric solid solutions of dilute magnetic oxides are mainly paramagnetic ${ }^{13-16}$ and highly defective alloys present room temperature ferromagnetism. ${ }^{17-19}$ In recent papers, ${ }^{20,21}$ a possible scenario for the origin of oxide ferromagnetism was presented. In contrast to the assumption that the entire sample is ferromagnetic, it is suggested that only small regions that contain defects such as grain boundaries become ferromagnetic. In this case, the defect concentration would be the limiting factor for the appearance of magnetism rather than doping by magnetic ions. The defects produce an impurity band inside the oxide band gap, and a Stoner type criterion could be used

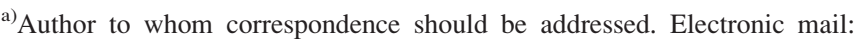
torres@fisica.unlp.edu.ar. Tel./Fax: +54 2214246062.
}

to predict magnetism. The dopants would contribute to this picture but not as the determining factor.

Mechanical alloying is a powerful technique to obtain non equilibrium phases. Repeated welding, fracturing, and re-welding of powder particles generate new surfaces, grain size reduction, and introduce a large number of defects. It is also possible to incorporate a higher iron concentration than with other solid state methods. Therefore, mechanically milled O-DMS materials are attractive systems to study the role of defects on the magnetic properties.

In previous works, we have reported results on Fe doped $\mathrm{TiO}_{2}$ samples obtained by mechanical alloying starting from $\alpha-\mathrm{Fe}_{2} \mathrm{O}_{3}$ and $\mathrm{TiO}_{2}$ powders (hematite series ${ }^{22,23}$ ) and from $\mathrm{FeO}$ and $\mathrm{TiO}_{2}$ samples (wustite series ${ }^{24}$ ). In this work, we present a comparative analysis of structural and magnetic properties of both samples series. We perform an extensive analysis of experimental results, and we find common characteristics that mainly depend on the relative fraction of $\mathrm{Fe}^{2+}$ and $\mathrm{Fe}^{3+}$. We find evidence that only a small fraction of the total volume of the sample, probably the grain boundaries, contributes to the ferromagnetic response. Ab initio calculations were performed in order to understand the role played by the concentration and distribution of both the Fe-dopants and the oxygen vacancies on the magnetic order. The calculations support the conclusion that oxygen vacancies are fundamental for the ferromagnetic response of $\mathrm{Fe}$-doped $\mathrm{TiO}_{2}$.

\section{EXPERIMENTAL METHODS}

Samples of $\mathrm{Ti}_{1-\mathrm{x}} \mathrm{Fe}_{\mathrm{x}} \mathrm{O}_{2-\delta}(2.5 \leq \mathrm{x} \leq 15$ at. \%) were prepared by mechanical alloying. Details of the sample 
preparation can be found in Refs. 22 and 24. The samples were characterized by X-ray diffraction (XRD), room temperature $\mathrm{X}$ ray absorption spectroscopy (XAS) at the $\mathrm{Fe}$ K-edge $(7112 \mathrm{eV}),{ }^{57} \mathrm{Fe}$ Mössbauer spectroscopy (MS), and magnetometric measurements using a commercial physical properties measurements system (PPMS, Quantum Design). The thermal dependence of the in-phase component of the ac-susceptibility was measured using a LakeShore 7130 ac-susceptometer. The alternating field was of $0.1 \mathrm{mT}$ at a frequency of $825 \mathrm{~Hz}$. Some of these data were previously published in Refs. 22 and 24 and are shown here for completeness.

\section{METHOD OF CALCULATION}

Spin-polarized electronic-structure calculations were performed with the $a b$ initio code Wien $2 \mathrm{~K},{ }^{25}$ which is an implementation of the full-potential Augmented Plane Wave plus local orbital method $\left(\mathrm{FP}-\mathrm{APW}+\mathrm{lo}^{26}\right)$. Exchange and correlation effects were treated within density-functional theory (DFT, Ref. 27) using the local spin density approximations (LSDA, Ref. 28). We also performed LDA $+U$ calculations $^{29}$ (with $U=5.0 \mathrm{eV}$ for the $\mathrm{Fe}$ and Ti-3d states).

In the APW+lo method, the unit-cell is divided into non-overlapping spheres with radius $R_{i}$ and an interstitial region. The parameter $R K_{M A X}$, which controls the size of the basis-set, was set to 6.0 ( $R$ is the smallest sphere radius and $K_{M A X}$ the largest wave number of the basis set). We also introduced local orbitals to include Ti- $3 s$ and $3 p, \mathrm{O}-2 s$ and $\mathrm{Fe}-3 p$ levels. Integration in the reciprocal space was performed using the tetrahedron method taking up to $100 k$ points in the first Brillouin zone. Atomic displacements around the impurities and oxygen vacancies have been obtained in the standard way described in Ref. 9.

\section{RESULTS}

\section{A. Structural characterization}

We checked by XRD, XAS, and MS that samples do not contain secondary ferromagnetic phases such as metallic iron or iron oxides. ${ }^{22,24}$ As an example, Fig. 1(a) shows the diffractogram of one of the samples with the corresponding fit performed with the Maud program. ${ }^{30}$ The obtained lattice parameters and average grain size (GS) are shown in Table I. In the hematite series, it is possible to see that the grain size changes only slightly with iron concentration whereas in the wustite series the average grain size increases from $13.8_{5}$ to $22.5_{7} \mathrm{~nm}$ with the iron concentration. In both series, the cell volume increases with increasing $\mathrm{Fe}$ concentration (see Fig. 1(b)) due to the incorporation of $\mathrm{Fe}^{3+}$ and/or $\mathrm{Fe}^{2+}$ and the consequent generation of oxygen vacancies. The increment in the cell volume can be expected from the fact that $\mathrm{TiO}_{2}$ is a mainly ionic crystal. When an oxygen vacancy is produced the Ti nearest neighbors of the oxygen vacancy repel each other, leaving a big hole at the vacancy site (see Ref. 31). This structural distortion can be the source of the increased volume of the cell.

Room temperature Mössbauer spectra (published in Refs. 23 and 24) show only paramagnetic interactions, one of
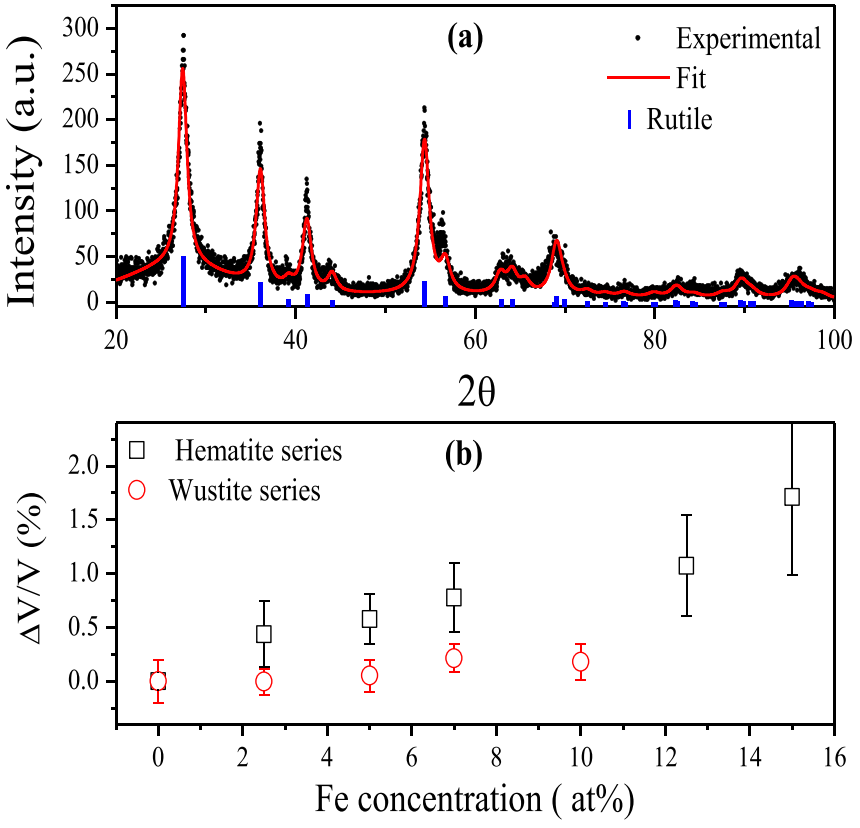

FIG. 1. (a) XRD pattern of 2.5 at $\%$ Fe-doped sample (wustite series) and the corresponding fit. Vertical lines indicate the reflections corresponding to rutile- $\mathrm{TiO}_{2}$ phase. (b) Relative volume change as a function of iron concentration for both series.

them corresponding to $\mathrm{Fe}^{2+}$ (isomer shift $(\delta)$ in the order of $1.0 \mathrm{~mm} / \mathrm{s}$, quadrupole splitting $(\Delta Q)$ in the order of $2.1 \mathrm{~mm} / \mathrm{s}$ ), and the other corresponding to $\mathrm{Fe}^{3+}(\delta \sim 0.4 \mathrm{~mm} / \mathrm{s}$, $\Delta \mathrm{Q} \sim 0.6 \mathrm{~mm} / \mathrm{s})$. In Table II, we show the relative fractions of $\mathrm{Fe}^{2+}$ and $\mathrm{Fe}^{3+}$. The contribution of $\mathrm{Fe}^{3+}$ was identified as $\mathrm{Fe}$ ions substituting for Ti with one oxygen vacancy near neighbor. ${ }^{32}$ The one corresponding to $\mathrm{Fe}^{2+}$ was tentatively assigned to $\mathrm{Fe}$ ions with two oxygen vacancies near neighbors. It is

TABLE I. Average GS and lattice parameters ( $a$ and $c$ ) obtained from the fits of $\mathrm{Ti}_{1-\mathrm{x}} \mathrm{Fe}_{\mathrm{x}} \mathrm{O}_{2}$ diffractograms.

\begin{tabular}{lccccccc}
\hline \hline & \multicolumn{3}{c}{ Hematite series } & & \multicolumn{3}{c}{ Wustite series } \\
\cline { 2 - 4 } \cline { 6 - 8 } at. \% Fe & GS (nm) & $a(\AA)$ & $c(\AA)$ & & GS (nm) & $a(\AA)$ & $c(\AA)$ \\
\hline 0.0 & $\ldots$ & $4.593_{6}$ & $2.958_{7}$ & $\ldots$ & $4.593_{6}$ & $2.958_{7}$ \\
2.5 & $13.9_{5}$ & $4.603_{4}$ & $2.958_{2}$ & & $13.8_{5}$ & $4.594_{7}$ & $2.956_{3}$ \\
5.0 & $11.4_{5}$ & $4.607_{3}$ & $2.957_{3}$ & & $15.2_{4}$ & $4.591_{3}$ & $2.962_{4}$ \\
7.0 & $11.2_{5}$ & $4.617_{3}$ & $2.950_{2}$ & & $18.6_{5}$ & $4.590_{4}$ & $2.967_{3}$ \\
10.0 & $12.2_{4}$ & $4.619_{3}$ & $2.952_{2}$ & & $22.5_{7}$ & $4.584_{2}$ & $2.974_{5}$ \\
12.5 & $12.9_{5}$ & $4.612_{4}$ & $2.965_{7}$ & & $\ldots$ & $\ldots$ & $\ldots$ \\
15.0 & $12.0_{5}$ & $4.636_{5}$ & $2.953_{2}$ & $\ldots$ & $\ldots$ & $\ldots$ \\
\hline \hline
\end{tabular}

TABLE II. Relative percentage area of $\mathrm{Fe}^{2+}( \pm 1 \%)$ obtained from the fits of the Mössbauer spectra for the $\mathrm{Ti}_{1-\mathrm{x}} \mathrm{Fe}_{\mathrm{x}} \mathrm{O}_{2}$ samples.

\begin{tabular}{lcc}
\hline \hline at $\% \mathrm{Fe}$ & Hematite series $\% \mathrm{Fe}^{2+}$ & Wustite series $\% \mathrm{Fe}^{2+}$ \\
\hline 2.5 & 70 & 0.0 \\
5.0 & 20 & 60 \\
7.0 & 0.0 & 73 \\
10.0 & 0.0 & 84 \\
12.5 & 44 & $\ldots$ \\
15.0 & 32 & $\ldots$ \\
\hline \hline
\end{tabular}



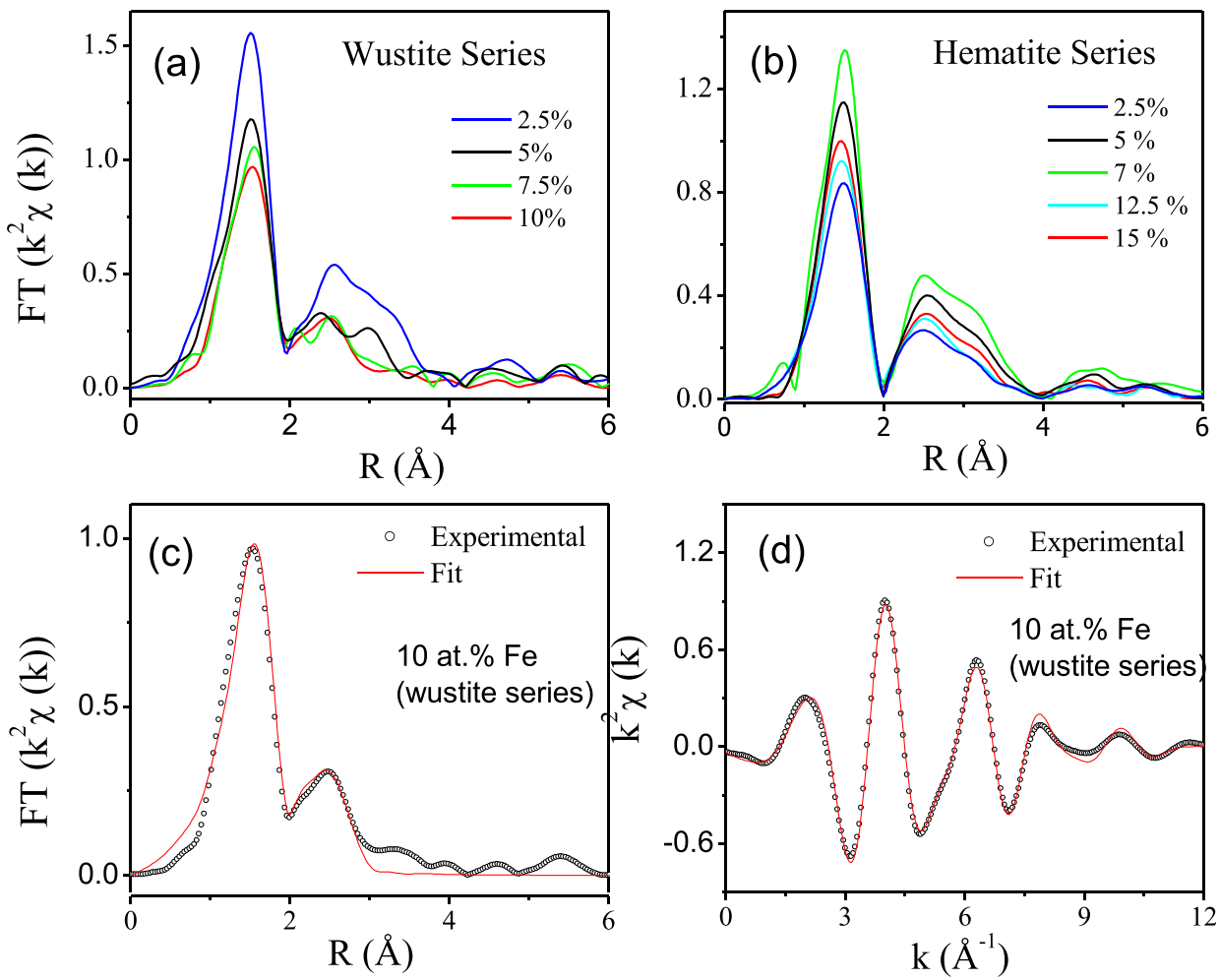

FIG. 2. FT of $\kappa^{2} \chi(\mathrm{k})$ obtained from $\mathrm{Ti}_{1-\mathrm{x}} \mathrm{Fe}_{\mathrm{X}} \mathrm{O}_{2}$ samples in the interval 2-11 $\AA^{-1}$ for Wustite series (a) and Hematite series (b). Experimental $\mathrm{k}^{2} \gamma(\mathrm{k})$ (c) and FT (d) corresponding to sample with 10 at.\% Fe (wustite series) and their corresponding fits. interesting to note that this component was observed only in samples obtained by mechanical milling, ${ }^{33,34}$ thin films, ${ }^{35}$ implanted monocrystals, ${ }^{36}$ or vacuum annealed samples ${ }^{37}$ but not in samples obtained by a chemical method. ${ }^{15}$ Another point to note is the absence of magnetic interactions in the Mössbauer spectra (sextets) even though there is a ferromagnetic-like contribution in the hysteresis loops (see below). This fact was also reported in other systems such as $\mathrm{Fe}$-doped $\mathrm{ZnO}^{38-40}$ and $\mathrm{Fe}$-doped $\mathrm{SnO}_{2}{ }^{41,42}$

Figures 2(a) and 2(b) shows a comparison of the Fourier transformed (FT) of the EXAFS (Extended X Ray Absorption Fine Structure) spectra corresponding to the two series of Fe-doped samples. The oscillations (extracted using Athena Code, Ref. 43) and their corresponding FT were fitted using Arthemis Code. ${ }^{43}$ In Figs. 2(c) and 2(d) the oscillation, the FT and the corresponding fitted curves for one of the samples are shown as an example. In the fit model we proposed two shells of Fe-neighbors (Fe-O and $\mathrm{Fe}-\mathrm{Ti}$ ) and kept a common value of $\sigma^{2}$ (Debye Waller factor) and $\Delta E$ (energy shift) for each series of samples. The amplitude reduction factor $S O^{2}$ (0.8) was determined experimentally from the spectrum of hematite. The corresponding bond lengths $(R)$, the coordination numbers for each layer (oxygen nearest neighbors (ONN) and TiNN) and Debye-Waller factors are reported in Table III.

As the bond lengths $\mathrm{Fe}-\mathrm{O}$ and $\mathrm{Fe}-\mathrm{Ti}$ are similar to those of $\mathrm{Ti}-\mathrm{O}$ and $\mathrm{Ti}-\mathrm{Ti}$ in rutile $\mathrm{TiO}_{2}$, we conclude that the Fe-dopants are substitutionally located at $\mathrm{Ti}$ sites in agreement with the Mössbauer results. In all cases the number of oxygen nearest neighbors is lower than six, indicating the presence of oxygen vacancies in the first coordination sphere of the iron ions.

In both series of samples, the oxygen coordination number clearly correlates with the relative fraction of $\mathrm{Fe}^{2+}$ (estimated from MS): the number of ONN of an iron ion decreases when the $\mathrm{Fe}^{2+}$ relative fraction increases (see Fig. 3(a)). In samples with a high concentration of $\mathrm{Fe}^{2+}$ the number of ONNs can be estimated at 4, while in samples where all the iron ions are in oxidation state $+3 \mathrm{ONN}=5.5$. The bond lengths $\mathrm{Fe}-\mathrm{O}$ increase with the fraction of $\mathrm{Fe}^{2+}$ (see Fig. 3(b)). This is consistent with the different ionic radii of $\mathrm{Fe}^{2+}\left(0.6358 \mathrm{pm}\right.$, coordination IV) and $\mathrm{Fe}^{3+}$ $(0.58 \mathrm{pm}$, coordination $\mathrm{V}){ }^{44}$ Based on a purely ionic model, when $\mathrm{Fe}^{2+}$ replaces a $\mathrm{Ti}^{4+}$ and there are no other $\mathrm{Fe}$ ions in the vicinity, one would expect to find an oxygen vacancy bound to it in order to neutralize the charge defect. However, when $\mathrm{Fe}^{3+}$ replaces $\mathrm{Ti}^{4+}$, charge neutrality is reached only when two iron ions share an oxygen vacancy. Our results

TABLE III. Fitting results of the first and second shells of neighbors of $\mathrm{Fe}$ ions compared to those of $\mathrm{Ti}$ in rutile $\mathrm{TiO}_{2}$. NN is the number of atoms located at a distance $\mathrm{R}$ from the central atom and $\sigma^{2}$ is the Debye Waller factor.

\begin{tabular}{|c|c|c|c|c|c|c|c|}
\hline \multirow[b]{2}{*}{ Series } & \multirow[b]{2}{*}{ Samples } & \multicolumn{3}{|c|}{ ONN } & \multicolumn{3}{|c|}{ TiNN } \\
\hline & & $\mathrm{NN}$ & $R(\AA)$ & $\sigma^{2}\left(\AA^{2}\right)$ & $\mathrm{NN}$ & $R(\AA)$ & $\sigma^{2}\left(\AA^{2}\right)$ \\
\hline & $\mathrm{TiO}_{2}$ & 4 & 1.937 & $\ldots$ & 4 & 3.039 & $\ldots$ \\
\hline & 2 & 1.964 & & & & & \\
\hline \multirow[t]{5}{*}{ Hematite } & $2.5 \%$ & $3.8_{3}$ & $2.037_{1}$ & $0.009_{1}$ & $2.3_{2}$ & $3.021_{3}$ & $0.016_{3}$ \\
\hline & $5.0 \%$ & $4.8_{3}$ & $1.996_{3}$ & $0.009_{1}$ & $3.3_{3}$ & $3.007_{9}$ & $0.016_{3}$ \\
\hline & $7.0 \%$ & $5.5_{2}$ & $1.986_{2}$ & $0.009_{1}$ & $3.8_{2}$ & $3.005_{3}$ & $0.016_{3}$ \\
\hline & $12.5 \%$ & $4.0_{2}$ & $2.010_{7}$ & $0.009_{1}$ & $2.7_{3}$ & $3.012_{8}$ & $0.016_{3}$ \\
\hline & $15.0 \%$ & $4.3_{3}$ & $2.009_{6}$ & $0.009_{1}$ & $2.8_{1}$ & $3.010_{7}$ & $0.016_{3}$ \\
\hline \multirow[t]{4}{*}{ Wustite } & $2.5 \%$ & $5.3_{2}$ & $1.985_{3}$ & $0.008_{2}$ & $3.7_{3}$ & $3.025_{5}$ & $0.015_{2}$ \\
\hline & $5 \%$ & $4.2_{3}$ & $1.993_{2}$ & $0.008_{2}$ & $2.8_{2}$ & $3.002_{3}$ & $0.015_{2}$ \\
\hline & $7.5 \%$ & $3.9_{2}$ & $2.040_{2}$ & $0.008_{2}$ & $3.0_{2}$ & $3.019_{6}$ & $0.015_{2}$ \\
\hline & $10 \%$ & $3.8_{2}$ & $2.051_{2}$ & $0.008_{2}$ & $3.2_{3}$ & $3.011_{4}$ & $0.015_{2}$ \\
\hline
\end{tabular}



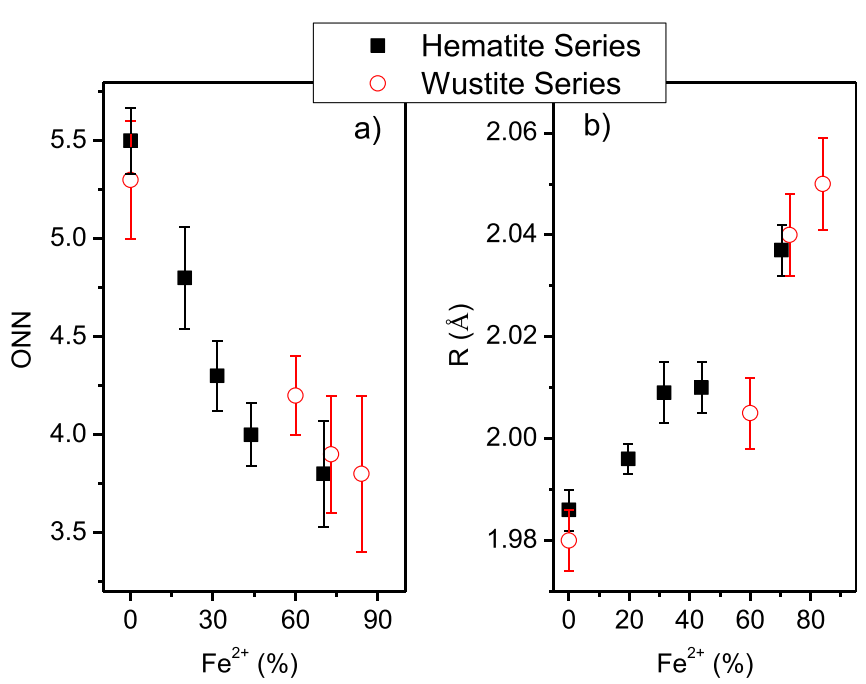

FIG. 3. (a) Dependence of ONN on $\mathrm{Fe}^{2+}$ at $\%$ for all samples. (b) $\mathrm{Fe}-\mathrm{O}$ distances in $\mathrm{Ti}_{1-x} \mathrm{Fe}_{\mathrm{x}} \mathrm{O}_{2}$ samples as a function of $\mathrm{Fe}^{2+}$ fraction.

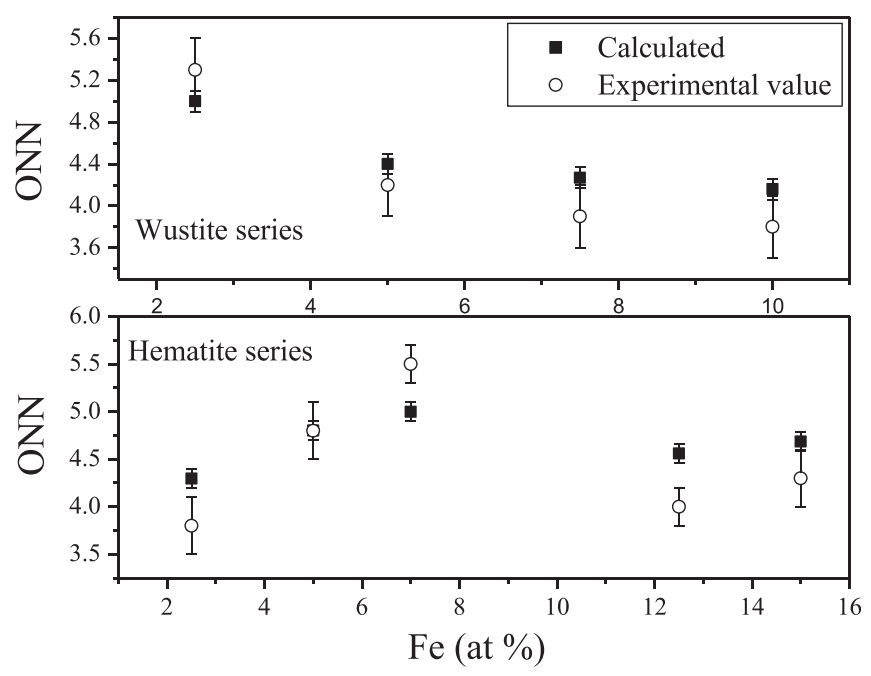

FIG. 4. Average number of oxygen near neighbor ONN of iron ion determined by EXAFS (experimental value) and the calculated ones taking into account $\mathrm{Fe}^{2+} / \mathrm{Fe}^{3+}$ ratio determined by Mössbauer spectroscopy assuming coordination four (two oxygen vacancies $\mathrm{NN}$ ) for $\mathrm{Fe}^{2+}$ and coordination five (one oxygen vacancy $\mathrm{NN}$ ) for $\mathrm{Fe}^{3+}$.

indicate that $\mathrm{Fe}^{2+}$ has coordination four (two oxygen vacancies nearest neighbors), and $\mathrm{Fe}^{3+}$ has coordination five (one oxygen vacancy nearest neighbors). We can also estimate the average number of oxygen nearest neighbor ions using the $\mathrm{Fe}^{2+} / \mathrm{Fe}^{3+}$ ratio determined by Mössbauer spectroscopy (see Figure 4). It can be seen that the calculated average number of oxygen agrees with the one determined by EXAFS. This implies that both $\mathrm{Fe}^{2+}$ and $\mathrm{Fe}^{3+}$ are coordinated with at least another iron ion forming dimers that share oxygen vacancies.

\section{B. Magnetic characterization}

The room temperature hysteresis loops (shown in Refs. 22 and 23) present two contributions: one of them ferromagnetic (FM) and the other paramagnetic (PM). The FM and PM components were fitted using the Stearns's function ${ }^{9}$ and a linear function, respectively. The obtained values of the saturation magnetization ( $M s$, in units of emu/g sample) and coercive field $(\mathrm{Hc})$ of the FM component and the magnetic susceptibility $(\chi)$ associated to the PM one are shown in Table IV. $M_{S}$ remains almost constant or independent of the $\mathrm{Fe}$ concentration, indicating that only a fraction of the total volume of the sample is involved in the ferromagnetic response of the system (see Figure 5, top). There is only a slight increase of $M s$ with the increase of the $\mathrm{Fe}^{2+}$ relative fraction but samples without $\mathrm{Fe}^{2+}$ also present room temperature ferromagnetism. If we subtract the saturation magnetization value of samples that do not contain $\mathrm{Fe}^{2+}$ from that of the other samples, a linear dependence is obtained (see Figure 5, bottom). We therefore conclude that there is a component of $M s$ related to the $\mathrm{Fe}^{2+}$ relative fraction and another one (constant and independent of concentration) originated by another factor.

The magnetic moment per iron atom $(M)$ decreases as the dopant concentration increases (see Fig. 6), suggesting either an antiferromagnetic interaction (AFM) among iron ions or frustration. Similar results were reported in many others Fe doped O-DMS. ${ }^{45-48}$ The fact that $M$ decreases with iron concentration rules out the possibility of ferromagnetism coming from iron precipitation. $M s$ is smaller than that expected for $\mathrm{Fe}^{2+}$ or $\mathrm{Fe}^{3+}$ indicating that only a small fraction of the Fe dopants participates in the observed ferromagnetism. Comparing samples with the same iron concentration the largest value of $M$ corresponds to the sample with a large fraction of $\mathrm{Fe}^{2+}$.

Although $\chi$ increases with the $\mathrm{Fe}$ concentration (Fig. 7(a)), its effective value per iron atom decreases (Fig. 7(b)). Considering that $M$ also decreases with the

TABLE IV. Saturation magnetization, Ms (emu/g); coercive field, Hc (T); paramagnetic susceptibility, $\chi$ (emu/Tg) and the magnetic moment per Fe atom, M $\left(\mu_{\mathrm{B}} /\right.$ at. Fe) obtained from the fits.

\begin{tabular}{|c|c|c|c|c|c|c|c|c|}
\hline \multirow[b]{2}{*}{$\begin{array}{l}\mathrm{Fe} \\
(\% \text { at. })\end{array}$} & \multicolumn{4}{|c|}{ Hematite series } & \multicolumn{4}{|c|}{ Wustite Series } \\
\hline & $\begin{array}{c}M s \\
(\mathrm{emu} / \mathrm{g})\end{array}$ & $\begin{array}{l}H c \\
(\mathrm{~T})\end{array}$ & $\begin{array}{c}M \\
\left(\mu_{\mathrm{B}} / \text { at. Fe }\right)\end{array}$ & $\begin{array}{c}\chi \\
(\mathrm{emu} / \mathrm{Tg})\end{array}$ & $\begin{array}{c}M s \\
(\mathrm{emu} / \mathrm{g})\end{array}$ & $\begin{array}{l}H c \\
(\mathrm{~T})\end{array}$ & $\begin{array}{c}M \\
\left(\mu_{\mathrm{B}} / \text { at. Fe }\right)\end{array}$ & $\begin{array}{c}\chi \\
(\mathrm{emu} / \mathrm{Tg})\end{array}$ \\
\hline 2.5 & $0.58_{3}$ & $0.0187_{5}$ & $0.33_{4}$ & $0.20_{3}$ & $0.22_{3}$ & $0.0140_{5}$ & $0.12_{1}$ & $0.09_{2}$ \\
\hline 5.0 & $0.49_{2}$ & $0.0152_{4}$ & $0.14_{2}$ & $0.10_{2}$ & $0.48_{5}$ & $0.0167_{7}$ & $0.13_{1}$ & $0.13_{3}$ \\
\hline 7.5 & $0.36_{4}$ & $0.0132_{4}$ & $0.07_{2}$ & $0.10_{2}$ & $0.44_{2}$ & $0.0196_{4}$ & $0.083_{4}$ & $0.17_{2}$ \\
\hline 10.0 & $0.29_{2}$ & $0.0059_{3}$ & $0.042_{2}$ & $0.13_{2}$ & $0.55_{4}$ & $0.0176_{6}$ & $0.079_{6}$ & $0.20_{3}$ \\
\hline 12.5 & $0.48_{3}$ & $0.0166_{7}$ & $0.06_{1}$ & $0.20_{3}$ & $\ldots$ & $\ldots$ & $\ldots$ & $\ldots$ \\
\hline 15.0 & $0.53_{5}$ & $0.0157_{5}$ & $0.05_{1}$ & $0.30_{4}$ & $\ldots$ & $\ldots$ & $\ldots$ & $\ldots$ \\
\hline
\end{tabular}




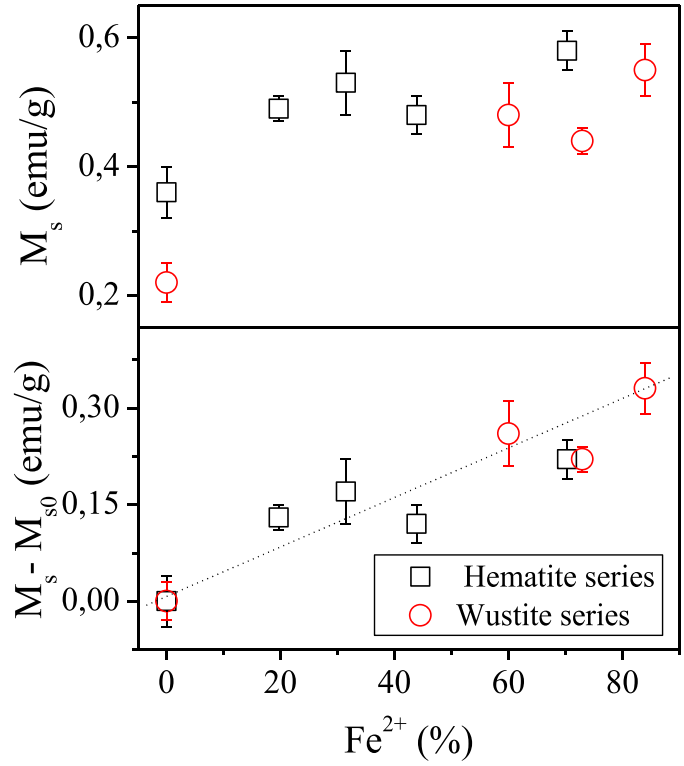

FIG. 5. (Top) $M_{s}$ as a function of $\mathrm{Fe}^{2+}$ fraction for hematite and wustite series, (Bottom) $M_{s}-M_{s O}$ values as a function of $\mathrm{Fe}^{2+}$ fraction.

concentration, this behavior must be related to an increase in the fraction of antiferromagnetically coupled atoms. A similar behavior was observed by us in Fe-doped $\mathrm{TiO}_{2}$ anatase nanoparticles obtained by chemical methods. ${ }^{13}$ In that system no ferromagnetic component was detected. Samples seemed to be purely paramagnetic, but the net magnetic moment per iron ion decreased with the temperature and the iron concentration due to the predominance of AFM interactions.

Figure 8 shows the thermal dependence of the in-phase component of the ac-susceptibility corresponding to samples of the wustite series. The curves corresponding to samples with smaller iron concentration present a similar dependence to that expected for a pure paramagnet but they do not tend to zero at high temperatures, indicating the presence of a fraction of ions in a magnetically ordered phase (ferromagnetic type). The in-phase susceptibility curves were fitted with the Curie-Weiss function

$$
\chi=\chi_{R T}+\frac{C}{(T-\Theta)}
$$

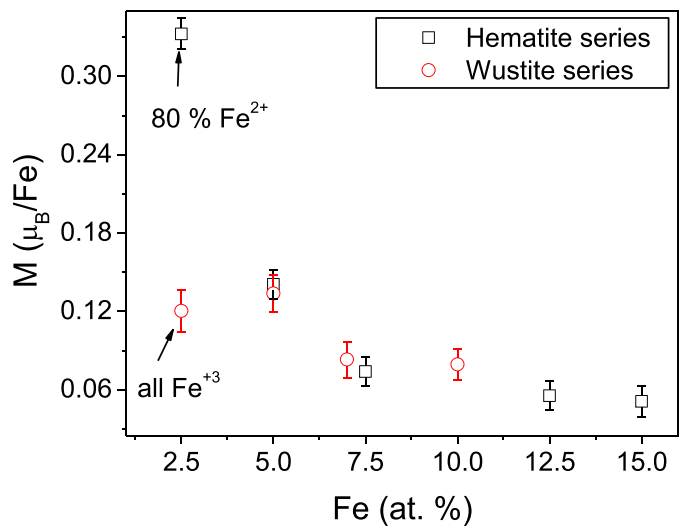

FIG. 6. Magnetic moment per Fe atoms as a function of Fe concentration for hematite and wustite series.

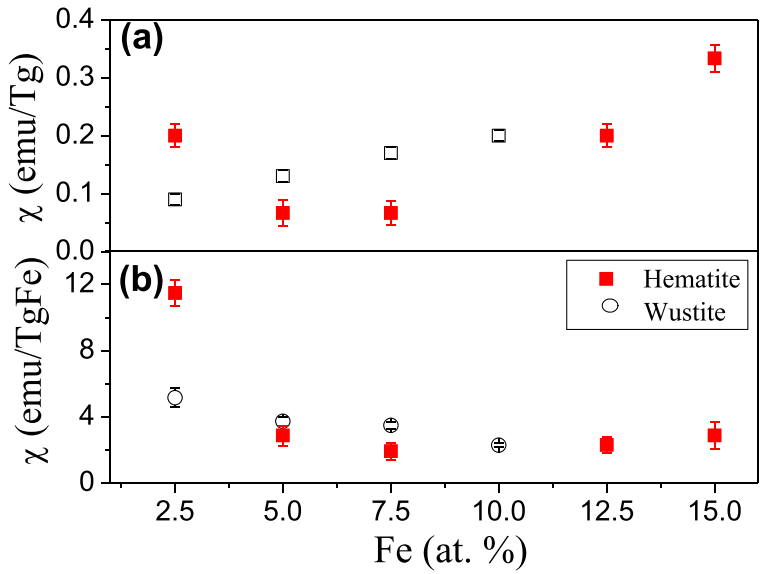

FIG. 7. (a) Susceptibility in units of emu/Tg (g, grams of samples) and (b) Susceptibility in unit of emu/TgFe (gFe: grams of iron).

where $\chi_{\mathrm{RT}}$ represents the ordered component at room temperature, $\mathrm{C}$ is the Curie constant $\left(C=\frac{n \mu_{0} \mu^{2}}{3 k_{B}}\right)$, and $\Theta$ is Curie-Weiss temperature. ${ }^{49}$ The values obtained for $\Theta$ were -1 and $-8 \mathrm{~K}$ for samples with 2.5 and 5 at. \% of iron, respectively. Negative values indicate the existence of AFM interactions between ions, which is stronger at higher iron concentrations.

\section{Ab-initio calculations}

The unit cell of rutile $\mathrm{TiO}_{2}$ is tetragonal ( $a=b=4.58451 \AA, c=2.9532 \AA$, space group $P 42 / \mathrm{mnm}^{50}$ In this structure, the $\mathrm{Ti}$ atoms are surrounded by a slightly distorted octahedron of oxygen atoms, with a rectangular basal plane (O1) at a distance of $1.94 \AA$ from Ti and two vertex atoms (O2) at $1.98 \AA$. In previous works (see Ref. 32 and references therein) we have simulated the doped systems considering periodically repeated supercells (SCs) in which some Ti-atoms are replaced by $\mathrm{Fe}$ in the rutile $\mathrm{TiO}_{2}$ host with and without oxygen vacancies. We found that when oxygen vacancies are introduced into the $\mathrm{TiO}_{2}$ structure along with iron impurities, the energetically preferred vacancy location is that of nearest neighbour to the impurity.

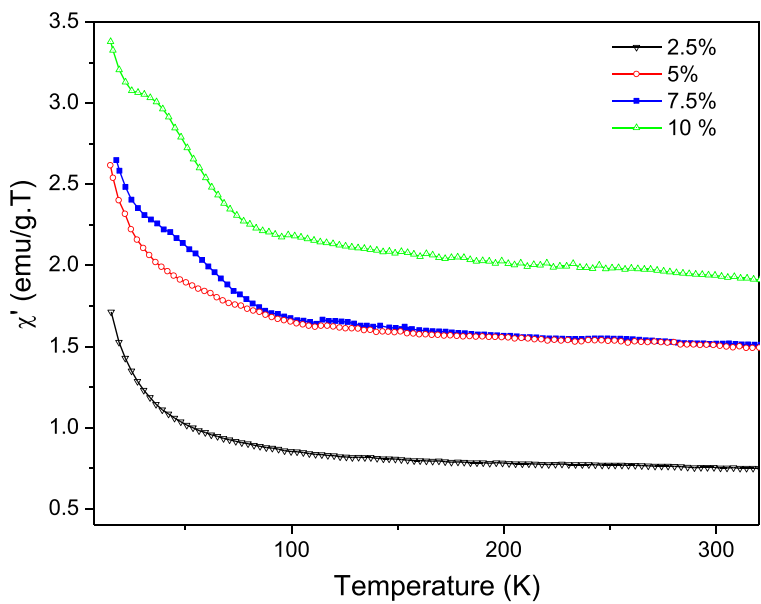

FIG. 8. In phase $\chi^{\prime}$ susceptibility of Wustite series samples measured at $825 \mathrm{~Hz}$. 
Additionally, we found that the strong interaction between oxygen vacancies and impurities increases the local magnetic moment at the Fe sites. Another important result was that Fe-doping lowered the formation energy of vacancies, so that doped systems will have more vacancies than the undoped ones. ${ }^{31,32}$ To be able to determine if the local magnetic moments of the Fe impurities align FM or AFM, we performed calculations with two $\mathrm{Fe}$ impurities in a 48-atom-SC for different impurity distributions (shown in Fig. 14, Ref. 32). We found that in absence of oxygen vacancies the most stable configuration was antiferromagnetic for the three configurations studied but when two substitutional Fe dopants were "linked" by one or two oxygen vacancies the ferromagnetic ordering was clearly preferred. The cluster of two Fe impurities linked by one oxygen vacancy can be described as a bound magnetic polaron (BMP, Ref. 51).

To explore the origin of the long-range ferromagnetic order, we considered two BMPs inside a larger SC of 108 atoms (see Figure 9(a)). We considered configuration $b$ in Fig. 14 of Ref. 32, with the oxygen vacancy in position b1.

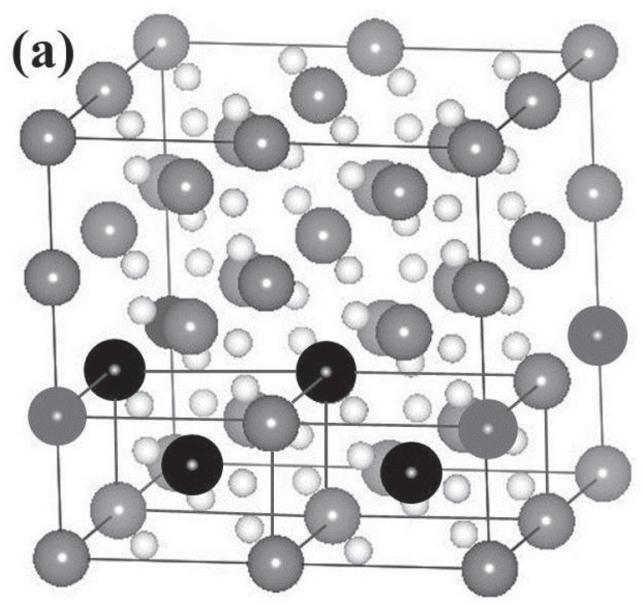

(b)

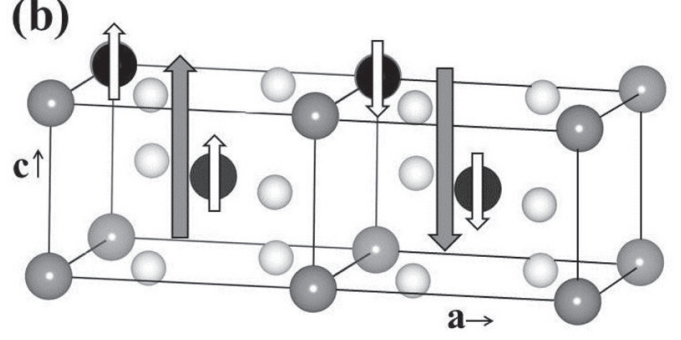

(c)

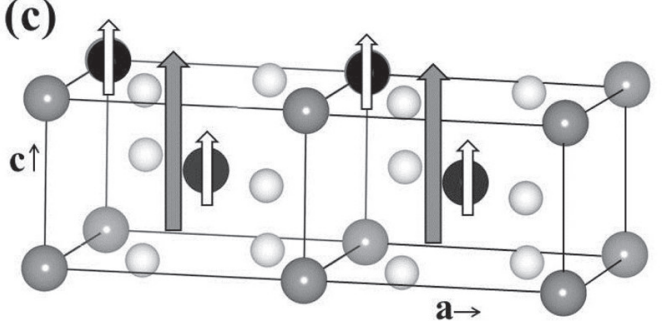

FIG. 9. (a) Supercell $2 \times 2 \times 3$ used to evaluate the coupling between BMP (Sn, gray balls; O, white balls; impurity, black balls), (b) antiferromagnetic, and (c) ferromagnetic configurations. In the case of no electron doping, $\Delta E=E_{\text {anti }}-E_{\text {ferro }}=-0.1 \mathrm{eV}$, antiferromagnetic alignment is favored. System doped with one electron $\Delta E=E_{\text {anti }}-E_{\text {ferro }}=-0.05 \mathrm{eV}$ ferromagnetic alignment is favored.
In this situation, the distance between the BMPs is $4.58 \AA$ Á. Two possible BMP alignments were considered, shown in Figs. 9(b) and 9(c). We obtained that antiferromagnetic alignment is favored by $0.1 \mathrm{eV}$, giving rise to a zero net magnetic moment. Thus, although an increase in iron concentration would favor a neighboring situation with an increase in the number of BMP, the antiferromagnetic BMP alignment would reduce the magnetization, a fact that is in very good agreement with the experimental results. Nevertheless, the origin of the ferromagnetic contribution is still unexplained and another mechanism must be considered to explain our results.

In our samples, the ferromagnetic saturation magnetization is nearly independent of the $\mathrm{Fe}$ concentration (only slightly increases with the increase of $\mathrm{Fe}^{2+}$ ), as was also observed in thin films. ${ }^{52}$ This is in agreement with the assumption that only a fraction of the Fe dopants are involved in the ferromagnetic response of the system. This fraction could be associated with dopants located at the grain boundary regions as previously observed in $\mathrm{Mn}$ - doped $\mathrm{ZnO}^{53}$ and $\mathrm{Co}-$ or Cr-doped $\mathrm{TiO}_{2}$ nanocrystals. ${ }^{54}$ It is well known that grain boundaries could act as trapping centers. For example in donor-doped $\mathrm{ZnO}$ and $\mathrm{BaTiO}_{3}$ ceramic, ${ }^{55,56}$ negatively charged grain boundary states compensate the positively charged donor centers in the bulk near the grain boundaries and form a depletion space charge layer. This led us to study the effect of extra electron doping, by adding one electron to the SC of Fig. 9(a) or replacing Ti by a donor impurity (such as $\mathrm{Ta}^{5+}$ ). For both approaches we found that the ferromagnetic coupling between BMP is the most stable configuration by $0.05 \mathrm{eV}$. This result shows that electron doping plays a fundamental role in mediating the nonlocal magnetic coupling between two BMP.

\section{DISCUSSION AND CONCLUSIONS}

Based on our experimental results, we find that by mechanical alloying we can obtain samples in which Fe ions are mainly incorporated into the $\mathrm{TiO}_{2}$ rutile structure with mixed valence states $(+2$ and +3$)$. EXAFS results show that iron coordination is similar to that of titanium in rutile but with oxygen vacancies close to the iron impurity in order to neutralize the charge defect induced by $\mathrm{Fe}^{2+}$ or $\mathrm{Fe}^{3+}$ substituting $\mathrm{Ti}^{4+}$. An increase in the concentration of oxygen vacancies is therefore predictable with an increase of the fraction of $\mathrm{Fe}^{2+} \cdot \mathrm{Fe}^{2+}$ and $\mathrm{Fe}^{3+}$ ions form dimers that share oxygen vacancies in agreement with our $a b$ initio calculations. These calculations predict that it is energetically favorable for oxygen vacancies to be formed in $\mathrm{Fe}-\mathrm{O}-\mathrm{Fe}$ paths.

Experimental results show that all samples present ferromagnetic, paramagnetic and antiferromagnetic contributions. The paramagnetic component was attributed to isolated iron ions and its contribution decreases when the iron concentration increases.

The negative values obtained for the Curie-Weiss temperature from ac-susceptibility measurements indicate the existence of antiferromagnetic interactions between $\mathrm{Fe}$ ions that are stronger at higher iron concentration. The distributed hyperfine magnetic interaction observed in Mössbauer 
measurements ${ }^{23}$ at low temperature $(4.2 \mathrm{~K})$ was associated with iron atoms coupled antiferromagnetically because this contribution grows when the iron concentration increases.

The ferromagnetic component is the minority one in all the cases. The saturation magnetization slightly changes with temperature, but the coercivity increases when temperature decreases. This component could not be related with a ferrimagnetic component, because in that case we would expect a maximum of saturation for samples with around $50 \%$ of $\mathrm{Fe}^{2+}$ and we did not observe such behavior. Nor ferromagnetism can be attributed to ferromagnetic phase precipitates.

Although the saturation magnetization increases with the fraction of $\mathrm{Fe}^{2+}$ (or oxygen vacancies), the magnetic moment per iron atom decreases with iron concentration indicating that the antiferromagnetic interaction between iron neighbors predominates over the effect of oxygen vacancies when iron concentration increases.

Taking into account our experimental and theoretical results, we present the following scenario. Two magnetic ions are coupled ferromagnetically when they are mediated by an oxygen vacancy forming a BMP. In cases where there are no free carriers, BMPs are coupled antiferromagnetically. Carrier doping mediates the ferromagnetic alignment between BMPs. We believe that itinerant carriers are confined to small regions, possibly grain boundaries, giving rise to a ferromagnetic ordering in such regions. In other regions there are oxygen vacancies but not itinerant carriers, resulting in antiferromagnetic alignment between BMPs and paramagnetic behavior of isolated ions. Therefore, oxygen vacancies would be responsible for forming BMPs, and free carriers for inducing the ferromagnetic coupling between BMPs.

Our results support the idea that ferromagnetism in Fe-doped rutile $\mathrm{TiO}_{2}$ strongly depends on defects. This fact explains the different experimental results for the magnetic moments and ferromagnetic response observed in samples prepared by different methods and/or different conditions reported in the literature. From our results, it is clear that the overall magnetic properties of $\mathrm{Fe}$-doped $\mathrm{TiO}_{2}$ are a statistical average of different defect configurations and only large scale simulations can reveal the complex behavior of reduced $\mathrm{Fe}$-doped $\mathrm{TiO}_{2}$ and reach a better understanding of the experimental results.

\section{ACKNOWLEDGMENTS}

This work was partially supported by Laboratorio Nacional de Luz Sincrotron (Campinas, Brasil), CONICET by Grant Nos. PIP 6005 and PIP 01111, National University of La Plata by el grant No. X543 and ANPCyT by Grant No. PICT 00898. The DC magnetic measurements were performed using the RN3M facilities.

\footnotetext{
${ }^{1}$ T. Dietl, J. Phys. Condens. Matter 19, 165204 (2007).

${ }^{2}$ J. M. D. Coey, Curr. Opin. Solid State Mater. Sci. 10, 83 (2006).

${ }^{3}$ C. Liu, F. Yun, and H. Morkoc, J. Mater. Sci.: Mater. Electron. 16, 555 (2005).

${ }^{4}$ T. Dietl, Nature Mater. 9, 965 (2010).
}

${ }^{5}$ T. Yu-Feng, H. Shu-Jun, Y. Shi-Shen, and M. Liang-Mo, Chin. Phys. B 22, 088505 (2013).

${ }^{6}$ B. Choudhury and A. Choudhury, J. Appl. Phys. 114, 203906 (2013).

${ }^{7}$ G. Cohen, V. Fleurov, and K. Kikoin, J. Appl. Phys. 101, 09H106 (2007).

${ }^{8}$ K. Griffin Roberts, M. Varela, S. Rashkeev, S. T. Pantelides, S. J. Pennycook, and K. M. Krishnan, Phys. Rev. B 78, 014409 (2008).

${ }^{9}$ S. Duhalde, M. F. Vignolo, F. Golmar, C. Chiliotte, C. E. Rodríguez Torres, L. A. Errico, A. F. Cabrera, M. Rentería, F. H. Sánchez, and M. Weissmann, Phys. Rev. B 72, 161313(R) (2005).

${ }^{10}$ A. N. Andriotis and M. Menon, Phys. Rev. B 87, 155309 (2013).

${ }^{11}$ D. H. Kim, S. I. Woo, S. H. Moon, H. D. Kim, B. Y. Kim, J. H. Cho, Y. G. Joh, and E. C. Kim, Solid State Commun. 136, 554 (2005).

${ }^{12}$ C. B. Fitzgerald, M. Venkatesan, A. P. Douvalis, S. Huber, J. M. D. Coey, and T. Bakas, J. Appl. Phys. 95, 7390 (2004).

${ }^{13}$ C. E. Rodríguez Torres, A. F. Cabrera, L. A. Errico, C. Adan, F. G. Requejo, M. Weissmann, and S. J. Stewart, J. Phys. Condens. Matter 20, 135210 (2008).

${ }^{14}$ H. B. de Carvalho, M. P. F. de Godoy, R. W. D. Paes, M. Mir, A. Ortiz de Zevallos, F. Iikawa, M. J. S. P. Brasil, V. A. Chitta, W. B. Ferraz, M. A. Boselli, and A. C. S. Sabioni, J. Appl. Phys. 108, 033914 (2010).

${ }^{15}$ L. Balcells, C. Frontera, F. Sandiumenge, A. Roig, B. Martínez, J. Kouam, and C. Monty, Appl. Phys. Lett. 89, 122501 (2006).

${ }^{16}$ A. Ney, K. Ollefs, S. Ye, T. Kammermeier, V. Ney, T. C. Kaspar, S. A. Chambers, F. Wilhelm, and A. Rogalev, Phys. Rev. Lett. 100, 157201 (2008).

${ }^{17}$ T. C. Kaspar, S. M. Heald, C. M. Wang, J. D. Bryan, T. Droubay, V. Shutthanandan, S. Thevuthasan, D. E. McCready, A. J. Kellock, D. R. Gamelin, and S. A. Chambers, Phys. Rev. Lett. 95, 217203 (2005).

${ }^{18}$ X. F. Liu, W. M. Gong, J. Iqbal, B. He, and R. H. Yu, Thin Solid Films 517, 6091 (2009).

${ }^{19}$ C. Song, S. N. Pan, X. J. Liu, X. W. Li, F. Zeng, W. S. Yan, B. He, and F. Pan, J. Phys. Condens. Matter 19, 176229 (2007).

${ }^{20}$ D. M. Khalid, A. Setzer, M. Ziese, P. Esquinazi, D. Spemann, A. Pöppl, and E. Goering, Phys. Rev. B 81, 214414 (2010).

${ }^{21}$ E. J. M. D. Coey, P. Stamenov, R. D. Gunning, M. Venkatesan, and K. Paul, New J. Phys. 12, 053025 (2010).

${ }^{22}$ A. M. Mudarra Navarro, V. Bilovol, A. F. Cabrera, C. E. Rodríguez Torres, and F. H. Sánchez. Physica B 404, 2838 (2009).

${ }^{23}$ V. Bilovol, A. M. Mudarra Navarro, W. T. Herrera, D. R. Sánchez, E. M. Baggio-Saitovich, C. E. Rodríguez Torres, F. H. Sánchez, and A. F. Cabrera, Hyperfine Interact. 195, 155 (2010).

${ }^{24}$ A. M. Mudarra Navarro, V. Bilovol, A. F. Cabrera, and C. E. Rodríguez Torres, Physica B 407, 3225 (2012).

${ }^{25}$ P. Blaha, K. Schwarz, G. Madsen, D. Kvasnicka, and J. Luitz, WIEN2k, An Augmented Plane Wave Plus Local Orbitals Program for Calculating Crystal Properties (Karlheinz Schwarz, Technical Universität Wien, Austria, 1999).

${ }^{26}$ E. Sjöstedt, L. Nordström, and D. J. Singh, Solid State Commun. 114, 15 (2000); G. K. H. Madsen, P. Blaha, K. Schwarz, E. Sjöstedt, and L. Nordström, Phys. Rev. B 64, 195134 (2001). See also S. Cottenier, Density Functional Theory and the Family of (L)APW-Methods: A Stepby-Step Introduction (KU Leuven, Belgium, 2002).

${ }^{27}$ P. Hohenberg and W. Kohn, Phys. Rev. 136, B864 (1964); W. Kohn and L. J. Sham, ibid. 140, A1133 (1965).

${ }^{28}$ J. P. Perdew and Y. Wang, Phys. Rev. B 45, 13244 (1992).

${ }^{29}$ V. I. Anisimov, I. V. Solovyev, M. A. Korotin, M. T. Czyzyk, and G. A. Sawatzky, Phys. Rev. B 48, 16929 (1993); M. T. Czyzyk and G. A. Sawatzky, ibid. 49, 14211 (1994).

${ }^{30}$ L. Lutterotti, S. Matthies, and H. R. Wenk. IUCr: Newsletter of the CPD 21, 14 (1999); available at http://www.mx.iucr.org/iucr-top/comm/cpd/ Newsletters/no21may1999/art17/art17.htm.

${ }^{31}$ L. A. Errico, M. Rentería, and M. Weissmann, Phys. Rev. B 72, 184425 (2005).

${ }^{32}$ A. M. Mudarra Navarro, L. A. Errico, C. E. Rodríguez Torres, A. F. Cabrera, V. Bilovol, M. Rentería, and M. Weissmann, Rutile, Properties, Synthesis and Applications, Chapter: Ferromagnetism in Transition Metal Doped Rutile $\mathrm{TiO}_{2}$ (Nova Science Publishers, United States of America, 2011).

${ }^{33}$ P. Xiaoyan, J. Dongmei, L. Yan, and M. Xueming, J. Magn. Magn. Mater. 305, 388 (2006).

${ }^{34}$ L. Fan, J. Dongmei, L. Yan, and M. Xueming, Physica B 403, 2193 (2008).

${ }^{35}$ K. J. Kim, Y. R. Park, and J. Y. Park, J. Korean Phys. Soc. 48, 1422 (2006). 
${ }^{36}$ M. Guermazia, G. Marest, A. Perez, B. D. Sawicka, J. A. Sawicki, P. Thevenard, and T. Tyliszczak, Mater. Res. Bull. 18, 529 (1983).

${ }^{37}$ H. M. Lee and Ch. S. Kim, J. Appl. Phys. 101, 09H110 (2007).

${ }^{38}$ Y. Lin, D. Jiang, F. Lin, W. Shi, and X. Ma, J. Alloys Compd. 436, 30 (2007).

${ }^{39}$ P. K. Sharma, R. K. Dutta, A. C. Pandey, S. Layek, and H. C. Verma. J. Magn. Magn. Mater. 321, 2587 (2009).

${ }^{40}$ F. Lin, D. Jiang, and X. Ma, Physica B 405, 1466 (2010).

${ }^{41}$ A. F. Cabrera, A. M. Mudarra Navarro, C. E. Rodríguez Torres, and F. H. Sánchez, Physica B 398, 215 (2007).

${ }^{42}$ J. Sakuma, K. Nomura, C. Barrero, and M. Takeda. Thin Solid Films 515, 8653 (2007).

${ }^{43}$ B. Ravel and M. Newville, Phys. Scr. 2005, 1007.

${ }^{44}$ R. D. Shannon, Acta Cryst. A 32, 751 (1976).

${ }^{45}$ M. V. Limaye, S. B. Singh, R. Das, P. Poddar, and S. K. Kulkarni, J. Solid State Chem. 184, 391 (2011).

${ }^{46}$ B. B. Straumal, S. G. Protasova, A. A. Mazilkin, Th. Tietze, E. Goering, G. Schütz, P. B. Straumal, and B. Baretzky Beilstein, J. Nanotechnol. 4, 361 (2013).
${ }^{47}$ K. Dodge, J. Chess, J. Eixenberger, G. Alanko, Ch. B. Hanna, and A. Punnoose, J. Appl. Phys. 113, 17B504 (2013).

${ }^{48}$ G. L. Beausoleil II, A. Thurber, S. S. Rao1, G. Alanko, C. B. Hanna, and A. Punnoose, J. Appl. Phys. 111, 07B546 (2012).

${ }^{49} \mathrm{Ch}$. Kittel, Introduction to Solid State Physics, 7th ed. (Wiley, New York, 1996).

${ }^{50}$ J. Hill and C. J. Howard, J. Appl. Crystallogr. 20, 467 (1987).

${ }^{51}$ M. D. Coey, M. Venkatesan, and C. B. Fitzgerald, Nature Mater. 4, 173 (2005).

${ }^{52}$ J. M. D. Coey, J. Appl. Phys. 97, 10D313 (2005).

${ }^{53}$ B. B. Straumal, A. A. Mazilkin, S. G. Protasova, A. A. Myatiev, P. B. Straumal, G. Schütz, P. A. van Aken, E. Goering, and B. Baretzky, Phys. Rev. B 79, 205206 (2009)

${ }^{54}$ J. D. Bryan, S. A. Santangelo, S. C. Keveren, and D. R. Gamelin, J. Am. Chem. Soc. 127, 15568 (2005).

${ }^{55}$ M. A. Alim, S. Li, F. Liu, and P. Cheng, Phys. Status Solidi A 203, 410 (2006).

${ }^{56}$ M. Vollman and R. Waser, J. Am. Ceram. Soc. 77, 235 (1994). 